\title{
The primitive soluble permutation groups of degree less than 256
}

\author{
M.W. ShorT
}

Primitive permutation groups have long been objects of interest: in 1872 Jordan [5], listed them up to degree 17 (but made a number of omissions, as pointed out by Miller in several papers from 1894 to 1900). In 1970 the primitive permutation groups were still only known up to degree 20 . Sims [7] verified this list by machine and later extended it to degree 50. Harada and Yamaki [4] determined the primitive permutation groups of degree 64 that have soluble socle. More recently, Dixon and Mortimer [3] have determined the primitive permutation groups of degree less than 1000 that have insoluble socle. The central topic of this thesis is to determine and provide electronic access to the soluble primitive permutation groups of degree less than 256 . This is motivated partly by the work of Dixon and Mortimer and partly because of the anticipated usefulness of such a list in a soluble quotient algorithm currently under development.

The primitive soluble permutation groups are in one-to-one correspondence with the irreducible soluble linear groups over finite prime fields. There is a large body of theory due to Jordan [2] and Suprunenko [8] which describes the structures of maximal irreducible soluble subgroups of general linear groups over arbitrary fields (hereafter called JS-maximals). This theory is extended as necessary to construct explicitly the JS-maximals of $G L(n, p)$ for a variety of pairs $(n, p)$, where $p$ is prime.

The early chapters of the thesis examine in detail the JS-maximals of $G L(2, p)$. These investigations lead to a theorem which lists exactly one member of each conjugacy class of irreducible soluble subgroups of $G L(2, p)$.

The later chapters deal with the problem of listing the irreducible soluble subgroups of $G L(n, p)$ for $n>2$ and $p^{n}<256$. Some of these cases are dealt with by ad hoc arguments. A theorem is obtained for listing certain irreducible soluble subgroups of $G L(q, p)$, where $q$ is any prime.

The two remaining cases are $G L(6,2)$ and $G L(4,3)$ of which the latter is more difficult. The relevant lists for these groups are compiled by a combination of theory

Received 17 April 1991

Thesis submitted to Australian National University, October 1990. Degree approved February 1991. Supervisors: Dr L.G. Kovács and Dr M.F. Newman.

Copyright Clearance Centre, Inc. Serial-fee code: 0004-9729/91 \$A2.00+0.00. 
and computation. An algorithm for listing the irreducible subgroups of several of the JS-maximals is presented, and is implemented in the group theory system Cayley [1]. The irreducible subgroups of the remaining JS-maximals are determined by theoretical techniques similar to those developed for $G L(2, p)$.

The provision of electronic access to the list of groups obtained is discussed. There are 370 conjugacy classes of irreducible soluble subgroups of $G L(n, p)$ for $n>1, p$ prime, and $p^{n}<256$. A Cayley library [6] which provides access to one group from each such class is described. The library also contains on-line help and some procedures for manipulating the groups; in particular, there is a procedure for converting the groups to the primitive soluble permutation groups to which they correspond. The library will be released as part of Cayley Version 3.8.

The thesis concludes with a note on work in progress on the irreducible soluble subgroups of $G L(8,2)$ (that is, the primitive soluble permutation groups of degree 256). The preparation of a list of these groups will involve a refinement of the algorithm used for $G L(4,3)$ and $G L(6,2)$; the original algorithm is not feasible for $G L(8,2)$ because of the large order of one of the JS-maximals.

\section{REFERENCES}

[1] John J. Cannon, 'An introduction to the group theory language, Cayley', in Computational Group Theory, Editor Michael D. Atkinson, pp. 145-183 (Academic Press, London, 1984).

[2] J. Dieudonné, 'Notes sur les travaux de C. Jordan relatifs à la théorie des groupes finis', in Oeuvres de Camille Jordan, tome 1, pp. XVII-XLII (Gauthier-Villars, Paris, 1961).

[3] John D. Dixon and Brian Mortimer, 'The primitive permutation groups of degree less than 1000', Math. Proc. Cambridge Philos. Soc. 103 (1988), 213-238.

[4] Koichiro Harada and Hiroyoshi Yamaki, 'The irreducible subgroups of $G L_{n}(2)$ with $n \leqslant 6$ ', C. R. Math. Rep. Acad. Sci. Canada 1 (1979), 75-78.

[5] C. Jordan, 'Sur l'énumération des groupes primitifs pour les dix-sept premiers degrés', C. R. Acad. Sci. 75 (1872), 1754-1757.

[6] M. W. Short, 'A library of irreducible soluble linear groups', in The Cayley Bulletin no. 4, Editor John Cannon (Department of Pure Mathematics, University of Sydney) (to appear).

[7] Charles C. Sims, 'Computational methods in the study of permutation groups', in Computational Problems in Abstract Algebra, Editor John Leech, pp. 169-183 (Pergamon Press, Oxford, 1970).

[8] D. A. Suprunenko, Matrix Groups, Translations of Mathematical Monographs, vol. 45 (American Mathematical Society, Providence, 1976). 
Mathematics Programme

Murdoch University

Murdoch WA 6150

Australia 